MATHEMATICS OF COMPUTATION

Volume 84, Number 294, July 2015, Pages 1835-1860

S 0025-5718(2015)02928-7

Article electronically published on January 13, 2015

\title{
GENERALIZATION OF PADÉ APPROXIMATION FROM RATIONAL FUNCTIONS TO ARBITRARY ANALYTIC FUNCTIONS - THEORY
}

\author{
CAN EVREN YARMAN AND GARRET M. FLAGG
}

\begin{abstract}
The Padé approximation has a long and rich history of theory and application and is known to produce excellent local approximations. We present a method for extending the basic idea of Padé approximation, that of matching a prescribed number of terms in the Taylor series expansion of a given function using rational functions, to any arbitrary function holormorphic in a neighborhood of the Taylor series expansion point. We demonstrate that providing the flexibility of using other functions in a Padé-type approximation yields highly accurate approximations having additional desirable properties. Additional properties that can be preserved in our method include comparable asymptotic behavior to the function to be approximated, or the preservation of band-limitedness in the approximation.
\end{abstract}

\section{INTRODUCTION}

The efficient representation of a function $f$ is determined by a variety of practical and theoretical considerations. We develop a novel application of the approximate solution to the partial realization problem that allows us to construct highly accurate approximations of a function $f$ that is analytic in a neighborhood of zero by a function $g$, and also analytic in a neighborhood of zero. Reasons for utilizing the function $g$ in the approximation will vary. For example, $g$ may be easier to implement on a computer, or may already be implemented in some optimized fashion. Or, it may have other desirable properties that can be taken advantage of under various algebraic and calculus manipulations of the representation. For the purposes of our analysis, we assume that $f$ has the following integral representation in terms of $g$,

$$
f(x)=\int_{a}^{b} \rho(\omega) g(\omega x) d \omega, \quad x \in \mathbb{C},
$$

for some generalized function $\rho(\omega)$ that is absolutely integrable over the finite interval $[a, b]$. For a given $\epsilon>0$, we seek an approximation

$$
G_{M, \epsilon}(x)=\sum_{m=1}^{M} \alpha_{m} g\left(\gamma_{m} x\right)
$$

that generalizes the Padé approximation of type $[M-1 / M]$ to $f(\mathrm{x})$ in the sense that

$$
\left|f^{(n)}(0)-G_{M, \epsilon}^{(n)}(0)\right| \leq \epsilon
$$

Received by the editor June 13, 2013 and, in revised form, November 18, 2013.

2010 Mathematics Subject Classification. Primary 30E05; 32A17, 32A26, 65D15, 41A21; Secondary 94A12, 94A11, 32A27, 33C10, 65T99. 
for $n=0, \ldots, 2 N$ for some $M \leq N$. Approximations of this form exhibit several advantages over standard Padé approximation. If $g$ is chosen appropriately, the approximation will exhibit comparable asymptotic growth and decay properties to $f$ on all of $\mathbb{C}$. Unlike Padé approximation, approximation (2) also has the ability to preserve spectral properties of the function to be approximated, such as band-limitedness. Moreover, a single accurate implementation of the approximating function $g$ is all that is necessary to generate a variety of highly accurate approximations to various functions $f$. This is especially useful for the case of special functions, which are typically implemented in terms of specialized polynomial and Padé or optimal minimax approximations. In the next section, we show how to generalize the moment matching formulation of the Padé problem to accommodate any kernel $g(\omega x)$ that is analytic in a neighborhood of zero for which an integral representation of the function $f$ may exist. We then show how to construct the approximation $G_{M, \epsilon}(x)$ in Section 2.1. In Section 3 we present an error analysis for our method. Finally, in Section 4 several examples are given demonstrating our approximation method for zero order and first order Bessel functions, as well as related functions.

\section{PADÉ-TyPe APPRoXimation USING FUnCtions ANALYTIC IN A NEIGHBORHOOD OF ZERO}

Let $f$ and $g$ be functions analytic in a neighborhood of the origin. Using their power series expansion at $x=0$, we have

$$
\begin{aligned}
& f(x)=\sum_{n=0}^{\infty} f_{n} x^{n}, \\
& g(x)=\sum_{n=0}^{\infty} g_{n} x^{n},
\end{aligned}
$$

where $f_{n}=f^{(n)}(0) / n$ !, and $g_{n}=g^{(n)}(0) / n$ !. Throughout the remainder of the exposition, we will assume that the enumeration of the nonzero power series coefficients of $f$ and $g$ corresponds, so that on the subsequence of nonzero coefficients in both series ordered according to their corresponding powers in $x$ we have $\left|f_{n} / g_{n}\right|<\infty$. For example, if $f$ is an even function, this requires that $g$ is an even function also. Now consider the formal power series

$$
h(x)=\sum_{n=0}^{\infty} \frac{f_{n}}{g_{n}} x^{n} .
$$

Our method of approximation is closely connected with the Padé approximant to the function $h(x)$. The following proposition clarifies how $h(x)$ is connected with the integral representation of $f$ in terms of the function $g$, under the appropriate assumptions.

Proposition 1. An integral representation of the form (11) exists whenever the moment problem determined by the sequence

$$
h_{n}=\frac{f_{n}}{g_{n}}=\int_{a}^{b} \rho(\omega) \omega^{n} d \omega,
$$

$n \geq 0$ has a possibly nonunique solution $\rho(\omega)$. 
Proof. Observe that

$$
\begin{aligned}
\int_{a}^{b} \rho(\omega) g(\omega x) d \omega & =\int_{a}^{b} \rho(\omega) \sum_{n=0}^{\infty} g_{n} x^{n} \omega^{n} d \omega \\
& =\sum_{n=0}^{\infty} g_{n}\left(\int_{a}^{b} \rho(\omega) \omega^{n} d \omega\right) x^{n} \\
& =f(x) .
\end{aligned}
$$

In Section 4 we present several examples of special functions which satisfy Proposition 1. In any case, assume now for the sake of argument that for some given $f$ and $g$, there exists $\rho(\omega)$ solving the moment problem in (3) and that $\rho(\omega)$ is given by

$$
\rho(\omega)=\sum_{m=1}^{M} \alpha_{m} \delta\left(\gamma_{m}-x\right),
$$

where $\delta(x)$ denotes the Dirac measure. Since $g$ is assumed to be analytic in a neighborhood of zero, by the Cauchy integral formula, we may write

$$
f(x)=\frac{1}{2 \pi i} \int_{\Gamma} g(x z) \phi(z) d z,
$$

where $\phi(z)=\int_{a}^{b} \frac{\rho(\omega)}{z-\omega} d \omega$, and $\Gamma$ is a contour surrounding the region of analyticity of $g$ with winding number one. In this scenario, $\phi(z)$ is a rational function with simple poles $\gamma_{m} \in \mathbb{C}$, and residues $\alpha_{m} \in \mathbb{C}, m=1, \ldots, M$, that is,

$$
\phi(z)=\sum_{m=1}^{M} \frac{\alpha_{m}}{z-\gamma_{m}}, \quad z \in \mathbb{C},
$$

and (1) becomes

$$
f(x)=\sum_{m=1}^{M} \alpha_{m} g\left(\gamma_{m} x\right) .
$$

By a straightforward application of the residue theorem, we may then write the coefficients $f_{n}$ of the power series expansion of $f$ in terms of the coefficients $g_{n}$ in the power series expansion of $g$ as

$$
f_{n}=g_{n} \sum_{m=1}^{M} \alpha_{m} \gamma_{m}^{n}
$$

Then we can rewrite (3) as

$$
h_{n}=\frac{f_{n}}{g_{n}}=\sum_{m=1}^{M} \alpha_{m} \gamma_{m}^{n},
$$

and the moment problem becomes equivalent to determination of $\alpha_{m}$ and $\gamma_{m}$ from the sequence $h_{n}$. Thus, for the special case of an integral measure $\rho(\omega) d \omega$ that consists of point masses, constructing the approximation (2) amounts to determining the parameters $\alpha_{m}, \gamma_{m}$ that satisfy (9). In the next section, we show how to construct the solution to this problem based on well-known results on moment matching from rational interpolation theory. We then show how to extend the rational interpolation approach to the general case of an arbitrary integral measure 
in the integral representation (11). The resulting generalization is summarized in Algorithm 1.

2.1. Realization theory and an algorithm for Padé-type approximations using arbitrary analytic functions. As we have seen, for the special case of an integral measure $\rho(\omega) d \omega$ that consists of point masses, constructing the approximation (21) amounts to determining the parameters $\alpha_{m}, \gamma_{m}$ that satisfy (91). This problem is known as the realization problem in rational interpolation theory. See [13 15, 18, 20] and the references therein for a more detailed discussion of realization theory from the control-theoretic perspective. For our purposes we need only consider some of the fundamental concepts and results from of the theory.

Definition 1. A sequence $h_{n} \in \mathbb{C}$ is realizable if there exists a tuple of matrices $(\mathbf{A}, \mathbf{b}, \mathbf{c}), \mathbf{A} \in \mathbb{R}^{M \times M}, \mathbf{b} \in \mathbb{R}^{M \times 1}, \mathbf{c} \in \mathbb{R}^{1 \times M}$ such that

$$
h_{n}=\mathbf{c A}^{n-1} \mathbf{b}
$$

for all $n$. The order of the realization $(\mathbf{A}, \mathbf{b}, \mathbf{c})$ is the dimension of the column space of $\mathbf{A}$.

Under the assumption that $\mathbf{A}$ has simple eigenvalues, the condition for realizability is equivalent to condition (9), which can readily be seen by simply diagonalizing $\mathbf{V}^{-1} \mathbf{A V}=\boldsymbol{\Lambda}$, and associating the parameters $\gamma_{m}$ with the eigenvalues of $\mathbf{A}$ and $\alpha_{m}=(\mathbf{c V})^{T}(m) \cdot\left(\mathbf{V}^{-1} \mathbf{b}\right)(m), m=1, \ldots, M$. The realizability of the sequence $h_{n}$ and its corresponding realization are completely characterized by the properties of the Hankel matrix $[\mathcal{H}]_{k, j}=h_{k+j-1}, k, j=1, \ldots, \infty$ formed from the moments $h_{n}$, $n>0$. The following theorem summarizes the conditions under which a rational interpolant can be found that satisfies the moment matching conditions of (9).

\section{Theorem 1.}

(1) A sequence $h_{n}, n>0$ is realizable if and only if rank $\mathcal{H}=K<\infty$.

(2) Every realization must be of at least order $K$.

A proof of these results can be found in [6]. If the conditions of Theorem 1 are satisfied, there are a number of different algorithms, such as the Silverman realization algorithm [20] that construct the realization of the sequence directly from $\mathcal{H}$.

Generally speaking, the integral measure $\rho(\omega) d \omega$ in (11) need not consist of point masses. We may nevertheless form the sequence of moments $h_{n}=f_{n} / g_{n}$ from an a priori knowledge of the McLaurin series representations of $f$ and $g$. In this case, the sequence $h_{n}$ will not satisfy the conditions of Theorem 1 However, it is still possible to solve a relaxation of the realization problem (9). Rather than solve (9) exactly, we instead find $\alpha_{m}$ and $\gamma_{m}$, that satisfy

$$
\left|h_{n}-\sum_{m=1}^{M} \alpha_{m} \gamma_{m}^{n}\right|<\epsilon, \text { for } n=0, \ldots, 2 N
$$

for some chosen error tolerance $\epsilon>0$. This problem was originally addressed by Kung in [16], and later revisited by Beylkin and Monzon in [9, 10. The number of terms $M$ in the sum necessary to obtain the error tolerance $\epsilon$ is governed by the singular value decay of the Hankel matrix associated with the sequence $h_{n}$ $n=0, \ldots, 2 N$ (see Algorithm 1 below). In general, the singular values of this 
matrix exhibit exponential decay, and we achieve the error tolerance for $M \ll N$. Hence there is an implicit reduction step involved in solving (10), which greatly reduces the number of terms used in the representation of $G_{M, \epsilon}(x)$. There are several methods for approximately solving (10). Two versions of the classical control theory approach to the solution of (10) are given by Kung in [16, and Kung and Lin in [17]. A more recent approach to the solution of this problem is detailed in 9, 10. For the sake of completeness we briefly outline the approach of Kung given in [16] in steps (2)-(9) of Algorithm 1. The key step in forming the generalized Padé approximation, however, is in forming the moments $h_{n}=f_{n} / g_{n}$ from the McLaurin series of $f$ and $g$.

Algorithm 1 Construction of the generalized Padé approximant to $f$

Inputs: $f_{n}, g_{n}$ for $n=0, \ldots, 2 N$ and $\epsilon$

Outputs: $\alpha_{m}, \gamma_{m}$ for $m=1, \ldots, M$ and $M \leq N$

(1) $h_{n}=f_{n} / g_{n}$

(2) Form $\boldsymbol{H}_{N}$, the Hankel matrix of dimension $N+1$ from $h_{n}$, i.e.

$\left[\boldsymbol{H}_{N}\right]_{k l}=h_{k+l}$, for $k, l=0, \ldots, N$

(3) Compute the singular value decomposition (SVD) of the Hankel matrix $\boldsymbol{H}_{N}=\boldsymbol{U} \boldsymbol{\Sigma} \boldsymbol{V}^{*}$

(4) Choose $M \leq N$ so that $\epsilon^{2}>\sqrt{M} \sigma_{M+1}$

(5) $\tilde{\boldsymbol{U}}=\boldsymbol{U}(1: N-1,1: M), \hat{\boldsymbol{U}}(2: N, 1: M), \boldsymbol{\Sigma}_{M}=\operatorname{diag}\left(\sigma_{1}, \ldots, \sigma_{M}\right)$. Here $\boldsymbol{A}\left(R_{1}: R_{2}, C_{1}: C_{2}\right)$ denotes the matrix formed by the rows $R_{1}$ to $R_{2}$ and columns $C_{1}$ to $C_{2}$ of the matrix $\boldsymbol{A}$ and $\operatorname{diag}\left(\sigma_{1}, \ldots, \sigma_{M}\right)$ denotes the diagonal matrix with diagonal elements $\sigma_{1}, \ldots, \sigma_{M}$.

(6) Form $\boldsymbol{A}=\left(\tilde{\boldsymbol{U}} \boldsymbol{\Sigma}_{M}^{1 / 2}\right)^{\dagger}\left(\hat{\boldsymbol{U}} \boldsymbol{\Sigma}_{M}^{1 / 2}\right), \boldsymbol{b}=\boldsymbol{\Sigma}_{M}^{1 / 2} \boldsymbol{V}^{*}(1: M, 1), \boldsymbol{c}=\boldsymbol{U}(1,1: M) \boldsymbol{\Sigma}_{M}^{1 / 2}$, where $\boldsymbol{\Sigma}_{M}^{1 / 2}=\operatorname{diag}\left(\sqrt{\sigma_{1}}, \ldots, \sqrt{\sigma_{M}}\right)$

(7) Compute eigenvalue decomposition of $\boldsymbol{A}=\boldsymbol{W} \boldsymbol{\Lambda} \boldsymbol{W}^{-1}$, where $\boldsymbol{\Lambda}$ is the diagonal matrix with diagonal elements $\lambda(\boldsymbol{A})$ being the eigenvalue of the matrix A.

(8) $\gamma_{m} \leftarrow \lambda(\boldsymbol{A})$

(9) $\alpha_{m} \leftarrow\left(\mathbf{W}^{T} \mathbf{c}^{T}\right) \circ\left(\mathbf{W}^{-1} \mathbf{b}\right)$, where $\boldsymbol{a} \circ \boldsymbol{b}$ denotes the elementwise (Hadamard) product of vectors $\boldsymbol{a}$ and $\boldsymbol{b}$.

Return $\gamma_{m}, \alpha_{m}$

Theorem 2. Let problem (10) be solved using Algorithm 1. Then

$$
\sum_{n=1}^{2 N}\left|h_{n}-\sum_{m=1}^{M} \alpha_{m} \gamma_{m}^{n}\right|^{2}<\epsilon^{2}
$$

Proof. See [16].

Corollary 1. Let $\gamma_{m}, \alpha_{m}$ be computed from Algorithm 1, with an error tolerance $\tilde{\epsilon}$ satisfying $\tilde{\epsilon}<\frac{\epsilon}{\max g_{n}}$, and let $G_{M, \epsilon}(x)=\sum \alpha_{m} g\left(\gamma_{m} x\right)$. Then $G_{M, \epsilon}(x)$ satisfies the conditions

$$
\left|f^{(n)}(0)-G_{M, \epsilon}^{(n)}(0)\right| \leq \epsilon
$$

for $n=0, \ldots, 2 N$. 
Proof. Observe that for $n=0, \ldots, 2 N$,

$$
\begin{aligned}
\left|f^{(n)}(0)-G_{M, \epsilon}^{(n)}(0)\right| & =\left|f_{n}-g_{n}\left(\sum \alpha_{m} \gamma_{m}^{n}\right)\right| \\
& =\left|g_{n}\right|\left|h_{n}-\sum \alpha_{m} \gamma_{m}^{n}\right| . \text { From Theorem 2 } \\
& <\left|g_{n}\right| \tilde{\epsilon} \\
& <\epsilon .
\end{aligned}
$$

Given $f_{n}$ and $g_{n}$, for $n=0, \ldots, 2 N$, the computationally dominant steps of Algorithm 1 are steps (1), (3), (6) and (7). Computation of $h_{n}$ requires $2 N+1$ multiplications, thus the computational complexity of the first step is $\mathcal{O}(N)$. Formation of the square Hankel matrix $\boldsymbol{H}_{N}$ has no computational complexity. $\boldsymbol{H}_{N}$ is a square matrix of dimension $N+1$, therefore the computational complexity of its singular value decomposition is $\mathcal{O}\left((N+1)^{3}\right)$. The computational cost of the rest of the algorithm is dominated by the computation of pseudoinverse in step (6) and eigenvalue decomposition in step (7) which have computational complexities of $\mathcal{O}\left(M(N+1)^{2}\right)$ and $\mathcal{O}\left(M^{3}\right)$, respectively. By choice, $M \leq N$, therefore the computational complexity of the algorithm is dominated by the singular value decomposition in step (3) which is $\mathcal{O}\left(N^{3}\right)$.

2.2. Connection to Padé approximation. The connection between Padé approximations, Gaussian quadrature and rational approximation is well known and has been discussed in great detail; see [8] and [7] for a more comprehensive discussion of the results we present here. We represent these results here in their simplest form only to illustrate that Padé approximation may be thought of as a special case of the approach we have delineated for constructing the approximations $G_{M, \epsilon}(x)$. Let $f$ have the integral representation

$$
f(x)=\int_{a}^{b} \frac{\rho(\omega)}{1-x \omega} d \omega .
$$

By applying the $M$ point Gaussian quadrature $\left(\alpha_{m}, \gamma_{m}\right)$ to $f$ we have

$$
f(x)=\sum_{m=1}^{M} \frac{\alpha_{m}}{1-x \gamma_{m}}+\mathcal{O}\left(x^{2 M}\right)=H_{M}(x)+\mathcal{O}\left(x^{2 M}\right) .
$$

The rational function

$$
H_{M}(x)=\sum_{m=1}^{M} \frac{\alpha_{m}}{1-x \gamma_{m}}
$$

clearly gives the type $[M-1 / M]$ Padé approximation to $f$, by the uniqueness of the Padé approximation [8]. Moreover, expanding $H_{M}(x)$ in a geometric series for sufficiently small $x$ and equating coefficients in $f$ yields the conditions

$$
f_{n}=\sum_{m=1}^{M} \alpha_{m} \gamma_{m}^{n}, \text { for } n=0, \ldots, 2 M-1,
$$

and it is now clear that $1 / \gamma_{m}$ and $\alpha_{m}$ determine the poles and residues respectively of the Padé approximation. Provided the type $[M-1 / M]$ Padé approximation of $f$ is not degenerate, meaning one of its poles is at $\infty$, and hence one of the $\gamma_{m}=0$ for some $m$, then it possible to restrict the approximation problem we presented above to a sufficiently small neighborhood of zero and where the representation (11) 
expanded as a formal power series is valid. Assuming $f$ is actually holomorphic in this neighborhood of zero, we may again apply Cauchy's integral representation theorem and obtain

$$
f(x)=\int_{\Gamma} \frac{\phi(z)}{1-x z} d z
$$

for some close contour $\Gamma$ around zero, where $\phi(z)=\int_{a}^{b} \frac{\rho(\omega)}{z-\omega} d \omega$. Hence, $H_{M}(1 / z)$ is a rational approximation to the function $\phi(z)$ that determines $f$ for the particular case of the kernel $g(x z)=\frac{1}{1-x z}$.

2.3. Properties of the approximation $G_{M, \epsilon}(x)$. All the motivating examples in Section 4 can be summarized in the following theorem and corollary.

Theorem 3. Let

$$
f_{n}=g_{n} \sum_{m=1}^{M} \alpha_{m} \gamma_{m}^{n}
$$

for $M<\infty$. If

$$
f(x)=\sum_{n=0}^{\infty} f_{n} x^{n}, \quad g(x)=\sum_{n=0}^{\infty} g_{n} x^{n},
$$

then

$$
f(x)=\sum_{m=1}^{M} \alpha_{m} g\left(\gamma_{m} x\right) .
$$

Proof. Substituting (12) in (13) and interchanging the order of summations we get

$$
\begin{aligned}
f(x) & =\sum_{n=0}^{\infty} f_{n} x^{n} \\
& =\sum_{n=0}^{\infty}\left[g_{n} \sum_{m=1}^{M} \alpha_{m} \gamma_{m}^{n}\right] x^{n} \\
& =\sum_{n=0}^{\infty} g_{n} \sum_{m=1}^{M} \alpha_{m} \gamma_{m}^{n} x^{n} \\
& =\sum_{m=1}^{M} \alpha_{m} \sum_{n=0}^{\infty} g_{n}\left(\gamma_{m} x\right)^{n} \\
& =\sum_{m=1}^{M} \alpha_{m} g\left(\gamma_{m} x\right)
\end{aligned}
$$

\section{Corollary 2.}

1. Even functions:

Let $f(x)$ and $g(x)$ be even functions and

$$
f_{n}=g_{n} \sum_{m=1}^{M} \alpha_{m} \gamma_{m}^{n}
$$


for $M<\infty$. If

$$
f(x)=\sum_{n=0}^{\infty} f_{n} x^{2 n}, \quad g(x)=\sum_{n=0}^{\infty} g_{n} x^{2 n}
$$

are analytic functions, then

$$
f(x)=\sum_{m=1}^{M} \alpha_{m} g\left(\sqrt{\gamma_{m}} x\right)
$$

2. Odd functions:

Let $f(x)$ and $g(x)$ be odd functions and

$$
f_{n}=g_{n} \sum_{m=1}^{M} \alpha_{m} \gamma_{m}^{n}
$$

for $M<\infty$. If

$$
f(x)=\sum_{n=0}^{\infty} f_{n} x^{2 n+1}, \quad g(x)=\sum_{n=0}^{\infty} g_{n} x^{2 n+1}
$$

then

$$
f(x)=\sum_{m=1}^{M} \frac{\alpha_{m}}{\sqrt{\gamma_{m}}} g\left(\sqrt{\gamma_{m}} x\right)
$$

Proof. This is a direct consequence of application of Theorem 3 to $f(\sqrt{x})$ and $g(\sqrt{x})$ for even functions, and to $x^{-1 / 2} f(\sqrt{x})$ and $x^{-1 / 2} g(\sqrt{x})$ for odd functions.

Corollary 3. Let

$$
f_{n}=g_{n} \sum_{m=1}^{M} \alpha_{m} \gamma_{m}^{n}
$$

for $M<\infty$.

1. If

$$
f(x)=\sum_{n=0}^{\infty} f_{n} \beta^{n x}, \quad g(x)=\sum_{n=0}^{\infty} g_{n} \beta^{n x}
$$

for some $\beta \in \mathbb{C}$, then

$$
f(x)=\sum_{m=1}^{M} \alpha_{m} g\left(x+\log _{\beta}\left(\gamma_{m}\right)\right)
$$

2. If

$$
f(x)=\sum_{n=0}^{\infty} f_{n} \log _{\beta}^{n}(x), \quad g(x)=\sum_{n=0}^{\infty} g_{n} \log _{\beta}^{n}(x),
$$

then

$$
f(x)=\sum_{m=1}^{M} \alpha_{m} g\left(\log _{\beta}\left(x^{\gamma_{m}}\right)\right) .
$$


Proof. We proceed similarly to the proof of Theorem 3 ,

1. Substituting (12) in (18) we get

$$
\begin{aligned}
f(x) & =\sum_{n=0}^{\infty}\left[g_{n} \sum_{m=1}^{M} \alpha_{m} \gamma_{m}^{n}\right] \beta^{n x} \\
& =\sum_{n=0}^{\infty}\left[g_{n} \sum_{m=1}^{M} \alpha_{m} \beta^{\log _{\beta}\left(\gamma_{m}\right) n}\right] \beta^{n x} \\
& =\sum_{n=0}^{\infty} g_{n} \sum_{m=1}^{M} \alpha_{m} \beta^{\left[\log _{\beta}\left(\gamma_{m}\right)+x\right] n} \\
& =\sum_{m=1}^{M} \alpha_{m} \sum_{n=0}^{\infty} g_{n} \beta^{\left[\log _{\beta}\left(\gamma_{m}\right)+x\right] n} \\
& =\sum_{m=1}^{M} \alpha_{m} g\left(\log _{\beta}\left(\gamma_{m}\right)+x\right) .
\end{aligned}
$$

2. Subtituting (12) in (19) we get

$$
\begin{aligned}
f(x) & =\sum_{n=0}^{\infty}\left[g_{n} \sum_{m=1}^{M} \alpha_{m} \gamma_{m}^{n}\right] \log _{\beta}^{n}(x) \\
& =\sum_{m=1}^{M} \alpha_{m} \sum_{n=0}^{\infty} g_{n}\left[\gamma_{m} \log _{\beta}(x)\right]^{n} \\
& =\sum_{m=1}^{M} \alpha_{m} \sum_{n=0}^{\infty} g_{n} \log _{\beta}^{n}\left(x^{\gamma_{m}}\right) \\
& =\sum_{m=1}^{M} \alpha_{m} g\left(\log _{\beta}\left(x^{\gamma_{m}}\right)\right) .
\end{aligned}
$$

It should be noted that the examples in Section 4 can be considered as an application of Corollary 1.

\section{ERROR ANALYSIS}

A straightforward analysis of the error in the approximation $G_{M, \epsilon}(x)$ follows an approach very similar to the classical analysis of the error in Gaussian quadrature first introduced by Gauss himself [11,12]. Let

$$
f(x)=\int_{a}^{b} \rho(\omega) g(x \omega) d \omega
$$

and let $\Gamma$ be a closed contour surrounding, but not intersecting $[a, b]$. By Cauchy's Theorem we have that

$$
\begin{aligned}
f(x) & =\int_{\Gamma} g(x z) \int_{a}^{b} \frac{\rho(\omega)}{z-\omega} d \omega d z \\
& =\int_{\Gamma} g(x z) \phi(z) d z
\end{aligned}
$$


where $\phi(z)=\int_{a}^{b} \frac{\rho(\omega)}{z-\omega} d \omega$. An upper bound on the approximation error follows directly from (20). We summarize this bound in the following theorem.

Theorem 4. Let $g(x)$ be given and assume that $f(x)$ satisfies (20). Let $G_{M, \epsilon}(x)$ be the approximation to $f$ constructed using Algorithm 1, and define $R_{M}(z)=$ $\sum_{m=1}^{M} \frac{\alpha_{m}}{z-\gamma_{m}}$. If $\gamma_{m} \in[a, b]$, then

$$
\begin{aligned}
\left|f(x)-G_{M, \epsilon}(x)\right| & \leq\|g(x z)\|_{\Gamma} \int_{\Gamma}\left|\phi(z)-R_{M}(z) \| d z\right| \\
& \leq\|g(x z)\|_{\Gamma, \infty}\left\|\phi(z)-R_{M}(z)\right\|_{\Gamma, \infty}|\Gamma|,
\end{aligned}
$$

where $|\Gamma|$ is the arclength of $\Gamma$ and $\|\cdot\|_{\Gamma, \infty}$ is the supremum norm over the curve $\Gamma$.

Proof. Let $\tilde{\rho}(\omega)=\sum \alpha_{m} \delta\left(x-\gamma_{m}\right)$. Then

$$
\begin{aligned}
\left|f(x)-G_{M, \epsilon}(x)\right| & =\left|\int_{a}^{b}(\rho(\omega)-\tilde{\rho}(\omega)) g(x \omega) d \omega\right| \\
& =\left|\int_{\Gamma}\left(\phi(z)-R_{M}(z)\right) g(x z) d z\right| \\
& \leq|| g(x z)\left\|_{\Gamma, \infty}\right\| \phi(z)-R_{M}(z) \|_{\Gamma, \infty}|\Gamma| .
\end{aligned}
$$

For small $x$ this upper bound is reasonably accurate; however, if $g$ is entire and bounded on the real line, this upperbound becomes impractical for large $x$, since $g$ cannot be bounded on all of $\mathbb{C}$ unless it is constant, by Liouville's Theorem.

Frequently, the moments $h_{n}=f_{n} / g_{n}$ decay very rapidly. In this situation, the approximation of the moments in terms of the sum $\sum \alpha_{m} \gamma_{m}^{n}$ is highly accurate until the values of the $h_{n}^{\prime} s$ fall below the chosen error tolerance $\epsilon$, and that after this the inequality $0<f_{n} / g_{n} \leq\left|\sum \alpha_{m} \gamma_{m}^{n}\right|<\epsilon$ is satisfied for $n>2 N$, even though the relative error in the approximation of the moments may grow very large. In this situation, we have the following error bound.

Theorem 5. Let $G_{M, \epsilon}(x)$ be an approximation to $f$ constructed according to Algorithm 1 for a given $\epsilon>0$. Assume that for $n>2 N, 0<f_{n} / g_{n} \leq\left|\sum \alpha_{m} \gamma_{m}^{n}\right|<\epsilon$. Then

$$
\left|f(x)-G_{M, \epsilon}(x)\right|<\frac{2 \epsilon M_{g}(R)}{1-x / R}
$$

for all $|x|<R$, where $M_{g}(R)=\max |g(z)|$ s.t. $|z|=R$.

Proof. Define $h_{n}=f_{n} / g_{n}$. Using Cauchy's Inequality (equation (24) on page 122 of [3]):

$$
g_{n}=\frac{g^{(n)}(0)}{n !} \leq \frac{M_{g}(R)}{R^{n}}
$$


we have

$$
\begin{aligned}
\left|f(x)-\sum_{m=1}^{M} \alpha_{m} g\left(\gamma_{m} x\right)\right| & =\left|\sum_{n=0}^{\infty} f_{n} x^{n}-\sum_{n=0}^{\infty} g_{n}\left(\sum_{m=1}^{M} \alpha_{m} \gamma_{m}^{n}\right) x^{n}\right| \\
& =\left|\sum_{n=0}^{\infty} g_{n}\left(h_{n}-\sum_{m=1}^{M} \alpha_{m} \gamma_{m}^{n}\right) x^{n}\right| \\
& \leq \sum_{n=0}^{\infty}\left|g_{n}\right| x^{n}\left(h_{n}-\sum_{m=1}^{M} \alpha_{m} \gamma_{m}^{n}\right) \mid \\
& \leq M_{g}(R) \sum_{n=0}^{\infty}\left|\left(\frac{x}{R}\right)^{n}\left(h_{n}-\sum_{m=1}^{M} \alpha_{m} \gamma_{m}^{n}\right)\right| \\
& \leq 2 \epsilon M_{g}(R) \sum_{n=0}^{\infty}\left|\frac{x}{R}\right|^{n} \\
& \leq \frac{2 \epsilon M_{g}(R)}{1-|x| / R}
\end{aligned}
$$

Theorems 3 and 4 provide good estimates on the error for relatively small values of $x \in \mathbb{R}$, depending on how quickly $g(x)$ grows in $\mathbb{C}$. In most of our applications, we have chosen $g(x)$ which decay on the real line. Hence, for sufficiently large $x \in \mathbb{R}$ the envelope of the function ( $f$ or $g$ ) which decays most quickly provides an accurate estimate for the error.

Remark 1. We observe that the error bound given in Theorem 3 for the approximation error is very similar in nature to the bound given for the error in the Padé approximation of $f$ of type $[M-1 / M]$. Let $R_{[M-1 / M]}=P_{M-1} / Q_{M}$ denote this Padé approximation to $f$. Then the error in the Padé approximant is bounded by 7

$$
\left|f(x)-R_{[M / M-1]}(x)\right| \leq\left|\frac{x}{R}\right|^{2 M} \frac{M_{f}(R) M_{Q_{M}}(R)}{(1-|x| / R) Q_{M}(x)} .
$$

Here again, $M_{f}(R)=\max |f(z)|$ s.t. $|z|<R$. In the case of Theorem 3 , the value $\epsilon$ essentially replaces the zero of order $2 M$ and the growth in the error over the disc is governed by $g$, rather than $M_{f}(R) M_{Q_{M}}(R)$.

\section{Motivating examples}

In this section we motivate our method using zeroth and first order Bessel functions of the first kind. We compare approximations obtained by the proposed method to the special functions routines available in MATLAB which are based on [4, 5]. We choose $\epsilon=2 \times 10^{-16}$ and the corresponding $M$ are obtained by step 2 of Algorithm 1,We present the plots of the functions obtained by MATLAB's routines and proposed approximations, their spectra and the absolute errors between them. The spectra are computed by applying discrete Fourier transform to the finely sampled functions and their approximations.

4.1. Zeroth order Bessel function of the first kind: Discritization of the integral representation through rational approximation. In certain cases it is possible to obtain the expression for $\rho(\omega)$ explicitly. For example, consider 
$f(x)=J_{0}(x)$, the zeroth order Bessel function of the first kind whose integral representation is given by (see page 360, equation 9.1.20 in [1]):

$$
J_{0}(x)=\frac{2}{\pi} \int_{0}^{1} \frac{1}{\sqrt{1-\omega^{2}}} \cos (\omega x) d \omega .
$$

Then we can identify $g(x)=\cos (x)$ and $\rho(\omega)=1 / \sqrt{1-\omega^{2}}$. Substituting the Maclaurin series expansion of the cosine function,

$$
\cos (x)=\sum_{n=0}^{\infty} \frac{(-1)^{n}}{(2 n) !} x^{2 n}
$$

and in (21),

$$
J_{0}(x)=\frac{2}{\pi} \sum_{n=0}^{\infty}\left[\int_{0}^{1} \frac{\omega^{2 n}}{\sqrt{1-\omega^{2}}} d \omega\right] \frac{(-1)^{n}}{(2 n) !} x^{2 n} .
$$

Equating each term of the series with the Maclaurin series expansion of the Bessel function,

$$
J_{0}(x)=\sum_{n=0}^{\infty} \frac{(-1)^{n}}{\left(2^{n} n !\right)^{2}} x^{2 n}
$$

we obtain the moments of $1 / \sqrt{1-\omega^{2}}$ :

$$
\int_{0}^{1} \frac{\omega^{2 n}}{\sqrt{1-\omega^{2}}} d \omega=\frac{\pi}{2} \frac{(2 n) !}{\left(2^{n} n !\right)^{2}}, \quad n=0,1, \ldots
$$

Thus finding a rational approximation to $\rho(\omega)=1 / \sqrt{1-\omega^{2}} \approx \sum_{m=1}^{M} \alpha_{m}\left(1-\gamma_{m}\right)^{-1}$ is equivalent to solving the exponential sum problem

$$
\sum_{m=1}^{M} \alpha_{m} \gamma_{m}^{2 n} \approx \frac{(2 n) !}{\left(2^{n} n !\right)^{2}}, \quad n=0,1, \ldots
$$

which can be solved using methods for representation of Hankel matrices such as Algorithm 1. As a consequence, one can approximate $J_{0}(x)$ by

$$
J_{0}(x) \approx \sum_{m=1}^{M} \alpha_{m} \cos \left(\gamma_{m} x\right) .
$$

Solving the moment problem (24) we obtained $\left(\alpha_{m}, \gamma_{m}\right)$ in Table 1. The resulting approximation (25), however, is far away from capturing the asymptotic decay property of $J_{0}$ as shown in Figure 1. On the other hand, when compared to the Padé approximation of type [21/22] (see Appendix (A), (25) has higher accuracy within a larger vicinity of zero.

4.2. First order Bessel function of the first kind. Differentiating of (21), consider $f(x)=-J_{0}^{\prime}(x) / x$ and $g(x)=\operatorname{sinc}(x)$, where $\operatorname{sinc}(x)=\sin (x) / x$ with

$$
\frac{-J_{0}^{\prime}(x)}{x}=\frac{2}{\pi} \int_{0}^{1} \frac{\omega^{2}}{\sqrt{1-\omega^{2}}} \operatorname{sinc}(\omega x) d \omega,
$$


TABLE 1. $\left(\alpha_{m}, \gamma_{m}\right)$ used in approximation $J_{0}(x) \approx \sum_{m=1}^{M} \alpha_{m} \cos \left(\gamma_{m} x\right)$.

\begin{tabular}{|c|c|c|}
\hline$m$ & $\alpha_{m}$ & $\gamma_{m}$ \\
\hline \hline 1 & 0.095979940059502 & 0.075341246466459 \\
\hline 2 & 0.095527611429490 & 0.223963536779947 \\
\hline 3 & 0.094673439193724 & 0.366561355147991 \\
\hline 4 & 0.093509003417115 & 0.499604276462964 \\
\hline 5 & 0.092152134096905 & 0.620183569182778 \\
\hline 6 & 0.090730283353054 & 0.726085980014931 \\
\hline 7 & 0.089364971890681 & 0.815761292824275 \\
\hline 8 & 0.086182508112798 & 0.997711597528443 \\
\hline 9 & 0.086525014629190 & 0.979412714085655 \\
\hline 10 & 0.087195093889236 & 0.942866940445256 \\
\hline 11 & 0.088159999928306 & 0.888214991866514 \\
\hline
\end{tabular}

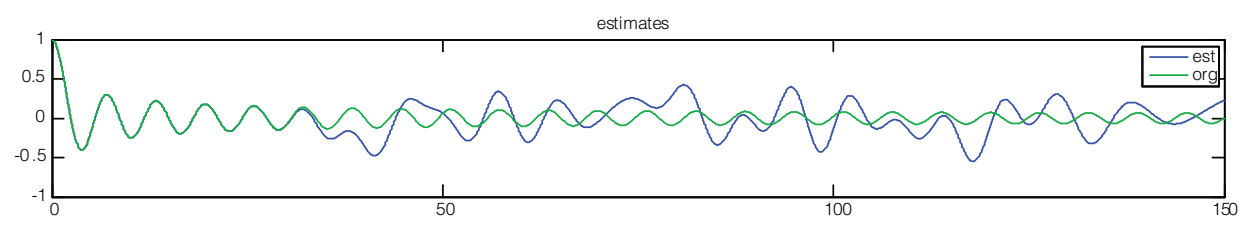

$\log _{10}$ absolute error
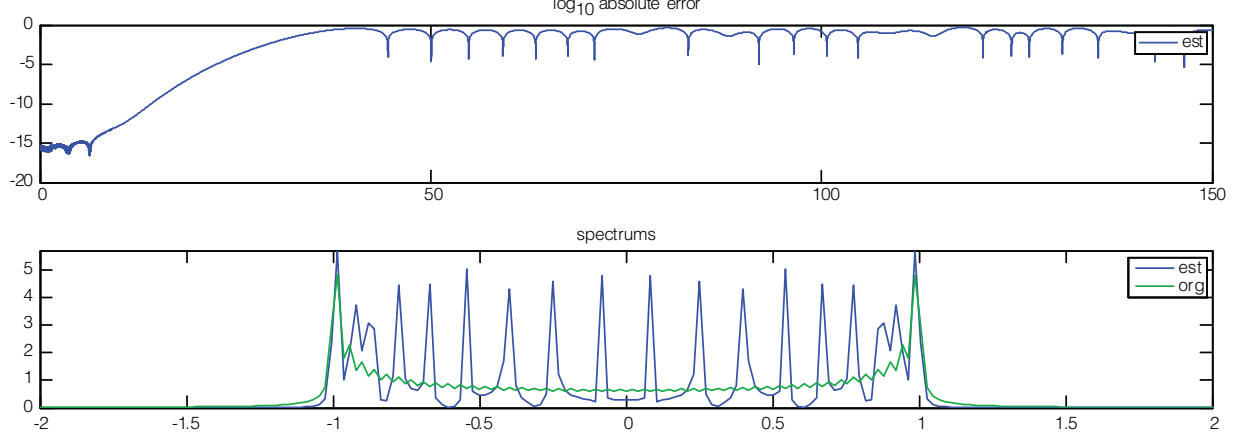

Figure 1. Approximation of the Bessel function $J_{0}(x)$ on $[0,150]$ (green) in terms of sum of cosines $J_{0}(x) \approx \sum_{m=1}^{M} \alpha_{m} \cos \left(\gamma_{m} x\right)$ (blue). The function and its approximation (top), corresponding logarithmic absolute error (middle) and spectra (bottom).

where we can identify $\rho(\omega)=\omega^{2} / \sqrt{1-\omega^{2}}$. Substituting the Maclaurin series expansion of $\operatorname{sinc}(x)$,

$$
\operatorname{sinc}(x)=\sum_{m=0}^{\infty} \frac{(-1)^{n}}{(2 n+1) !} x^{2 n},
$$

into (26) and equating it with the series representation

$$
\frac{-J_{0}^{\prime}(x)}{x}=\sum_{n=0}^{\infty} \frac{(-1)^{n} 2(n+1)}{\left(2^{n+1}(n+1) !\right)^{2}} x^{2 n},
$$


TABLE 2. Table of $\left(\alpha_{m}, \gamma_{m}\right)$ used in approximation $-J_{0}^{\prime}(x) / x \approx$ $\sum_{m=1}^{M} \alpha_{m} \operatorname{sinc}\left(\gamma_{m} x\right)$.

\begin{tabular}{|c|c|c|}
\hline$m$ & $\alpha_{m}$ & $\gamma_{m}$ \\
\hline \hline 1 & 0.071835875786124 & 0.998398980812295 \\
\hline 2 & 0.070105962819979 & 0.985607972315142 \\
\hline 3 & 0.066707566523699 & 0.960119491462000 \\
\hline 4 & 0.061763552087851 & 0.922131706810163 \\
\hline 5 & 0.055460542483329 & 0.871973945670952 \\
\hline 6 & 0.048057515622654 & 0.810154751809044 \\
\hline 7 & 0.039903785860314 & 0.737429599003079 \\
\hline 8 & 0.031461409389528 & 0.654848549013608 \\
\hline 9 & 0.001217711011290 & 0.124261388401203 \\
\hline 10 & 0.004615785021319 & 0.244670398855131 \\
\hline 11 & 0.023293297102112 & 0.563647668207971 \\
\hline 12 & 0.009649543856840 & 0.358509128042139 \\
\hline 13 & 0.015927452434960 & 0.464799923308326 \\
\hline
\end{tabular}

we obtain the moments

$$
\int_{0}^{1} \frac{\omega^{2(n+1)}}{\sqrt{1-\omega^{2}}} d \omega=\frac{\pi}{2} \frac{(2(n+1)) !}{\left(2^{(n+1)}(n+1) !\right)^{2}}, \quad n=0,1, \ldots,
$$

which leads to

$$
\sum_{m=1}^{M} \alpha_{m} \gamma_{m}^{2 n} \approx \frac{(2(n+1)) !}{\left(2^{(n+1)}(n+1) !\right)^{2}}, \quad n=0,1, \ldots
$$

As a consequence, one can approximate $-\frac{J_{0}^{\prime}(x)}{x}$ by

$$
-\frac{J_{0}^{\prime}(x)}{x} \approx \sum_{m=1}^{M} \alpha_{m} \operatorname{sinc}\left(\gamma_{m} x\right)
$$

The resulting approximation (29) using $\left(\alpha_{m}, \gamma_{m}\right)$ in Table 2 is presented in Figure 2 and it does capture the asymptotic decaying behavior of $-J_{0}^{\prime}(x) / x$.

Since the first order Bessel function is related to the zeroth order Bessel function of the first kind, we have

$$
\begin{aligned}
J_{1}(x) & =-J_{0}^{\prime}(x) \\
& =x \frac{2}{\pi} \int_{0}^{1} \frac{\omega^{2}}{\sqrt{1-\omega^{2}}} \operatorname{sinc}(\omega x) d \omega \\
& =\frac{2}{\pi} \int_{0}^{1} \frac{\omega}{\sqrt{1-\omega^{2}}} \sin (\omega x) d \omega \\
& \approx x \sum_{m=1}^{M} \alpha_{m} \operatorname{sinc}\left(\gamma_{m} x\right) \\
& \approx \sum_{m=1}^{M} \frac{\alpha_{m}}{\gamma_{m}} \sin \left(\gamma_{m} x\right) .
\end{aligned}
$$



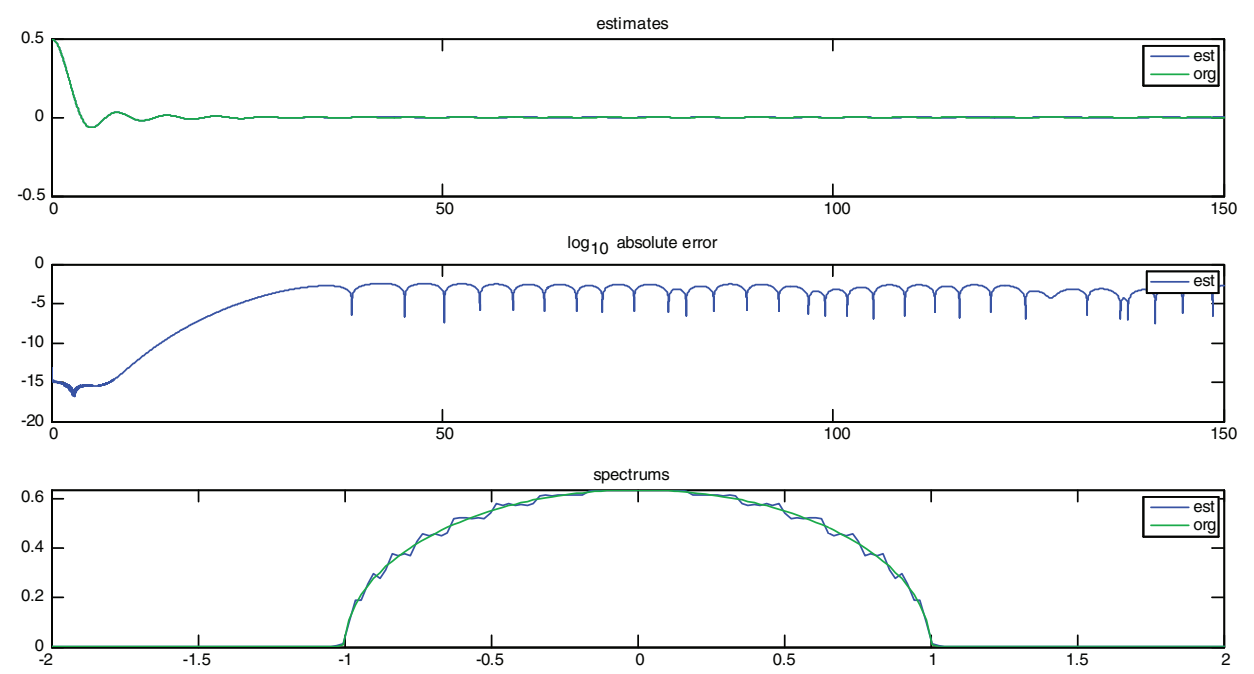

Figure 2. Approximation of the function $-J_{0}^{\prime}(x) / x$ on $[0,150]$ (green) in terms of sum of sincs $-J_{0}^{\prime}(x) / x \approx \sum_{m=1}^{M} \alpha_{m} \operatorname{sinc}\left(\gamma_{m} x\right)$ (blue). The function and its approximation (top), corresponding logarithmic absolute error (middle) and spectra (bottom).
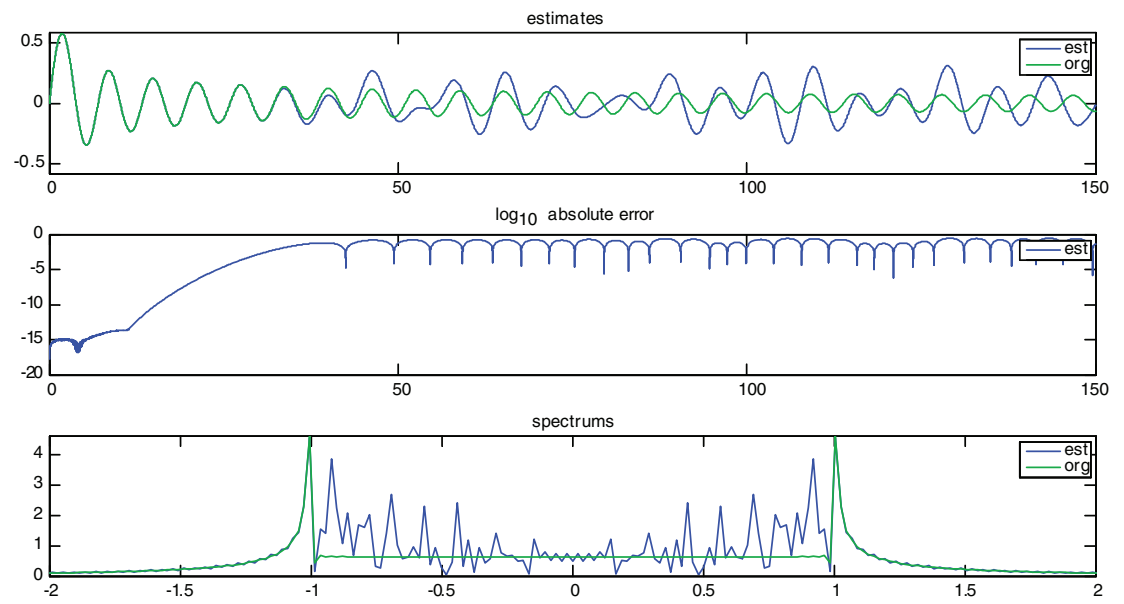

Figure 3. Approximation of the function $J_{1}(x)$ on $[0,150]$ (green) in terms of sum of sines $J_{1}(x) \approx \sum_{m=1}^{M} \frac{\alpha_{m}}{\gamma_{m}} \sin \left(\gamma_{m} x\right)$ (blue). The function and its approximation (top), corresponding logarithmic absolute error (middle) and spectra (bottom).

The resulting approximation (30), is presented in Figure 3. Similarly to the case of approximating $J_{0}(x)$ by (25), (30) doesn't capture the asymptotic decaying behavior of $J_{1}(x)$. 
TABLE 3. Table of $\left(\alpha_{m}, \gamma_{m}\right)$ used in approximation $J_{0}(x) \approx \sum_{m=1}^{M} \alpha_{m} \operatorname{sinc}\left(\gamma_{m} x\right)$.

\begin{tabular}{|c|c|c|}
\hline$m$ & $\alpha_{m}$ & $\gamma_{m}$ \\
\hline \hline 1 & -0.002287629127256 & 0.151888522515019 \\
\hline 2 & -0.009452739721483 & 0.299376714293969 \\
\hline 3 & -0.022545096665550 & 0.438465780256230 \\
\hline 4 & -0.043894368279012 & 0.565873165378772 \\
\hline 5 & -0.078425591443122 & 0.679158599794604 \\
\hline 6 & -0.137015048504309 & 0.776648319474267 \\
\hline 7 & 9.909619553181633 & 1.001322398612965 \\
\hline 8 & -6.657768151246734 & 0.992805795683471 \\
\hline 9 & -1.222883382024550 & 0.965595345971015 \\
\hline 10 & -0.246580111296777 & 0.857265389434489 \\
\hline 11 & -0.488767434872840 & 0.920364566217202 \\
\hline
\end{tabular}

\subsection{Zeroth and first order Bessel function of the first kind revisited.}

4.3.1. Zeroth order Bessel function of the first kind. In some cases, it is challenging to obtain an expression for $\rho(\omega)$, or at least in the sense of classical functions. For example, consider $f(x)=J_{0}(x)$ and $g(x)=\operatorname{sinc}(x)$ and the integral representation of the form (see Appendix B]

$$
J_{0}(x)=\frac{2}{\pi} \int_{0}^{1} \rho(\omega) \operatorname{sinc}(\omega x) d \omega,
$$

where

$$
\rho(\omega)=\frac{\omega^{2} \chi_{[-1,1]}(\omega)}{\left(1-\omega^{2}\right)^{3 / 2}}+\frac{\omega[\delta(\omega-1)-\delta(\omega+1)]}{\sqrt{1-\omega^{2}}}
$$

is a generalized function [19.

Through the Maclaurin series of $J_{0}(x)$ and $\operatorname{sinc}(x)$, one has the moments

$$
\frac{2}{\pi} \int_{0}^{1} \rho(\omega) \omega^{2 n} d \omega=\frac{(2 n+1) !}{\left(2^{n} n !\right)^{2}}, \quad n=0,1, \ldots
$$

Assuming the rational form (4) to approximate $\rho(\omega),\left(\alpha_{m}, \gamma_{m}\right)$ should satisfy

$$
\sum_{m=1}^{M} \alpha_{m} \gamma_{m}^{2 n} \approx \frac{(2 n+1) !}{\left(2^{n} n !\right)^{2}}, \quad n=0,1, \ldots
$$

As a consequence, one can approximate $J_{0}(x)$ by

$$
J_{0}(x) \approx \sum_{m=1}^{M} \alpha_{m} \operatorname{sinc}\left(\gamma_{m} x\right)
$$

The resulting approximation (32) using $\left(\alpha_{m}, \gamma_{m}\right)$ in Table 3 is presented in Figure 4 and, unlike approximation (25), it does capture the asymptotic decaying behavior of $J_{0}(x)$. When compared to the Padé approximation of type [21/22] (see Appendix (A), (32) has higher accuracy within a larger vicinity of zero as well as a decay rate that is more comparable to that of $J_{0}(x)$. 

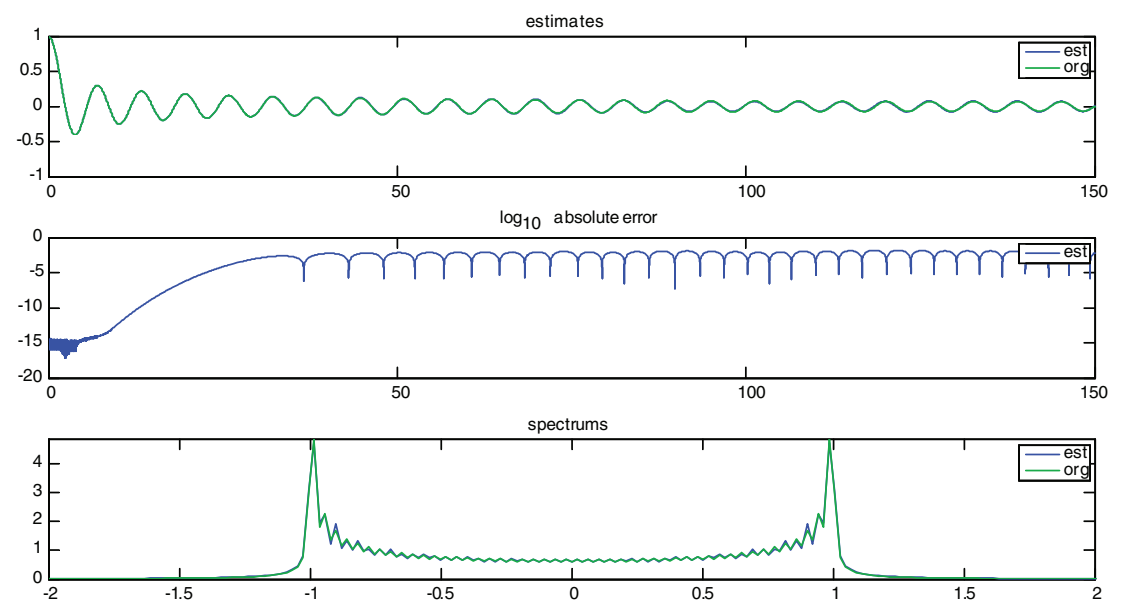

Figure 4. Approximation of the function $J_{0}(x)$ on $[0,150]$ (green) in terms of a sum of $\operatorname{sincs} J_{0}(x) \approx \sum_{m=1}^{M} \alpha_{m} \operatorname{sinc}\left(\gamma_{m} x\right)$ (blue). The function and its approximation (top), corresponding logarithmic absolute error (middle) and spectra (bottom).

4.3.2. First order Bessel function of the first kind. Recalling the relationship between $J_{1}(x)$ and $J_{0}(x)$, by (31), we can approximate $J_{1}(x)$ by

$$
\begin{aligned}
J_{1}(x) & =-J_{0}^{\prime}(x) \\
& =-\int \frac{2^{1}}{\pi} \rho(\omega) \operatorname{sinc}^{\prime}(\omega x) d \omega \\
& \approx-\sum_{m=1}^{M} \alpha_{m} \operatorname{sinc}^{\prime}\left(\gamma_{m} x\right) d \omega
\end{aligned}
$$

where

$$
\rho(\omega)=\frac{\omega^{3} \chi_{[-1,1]}(\omega)}{\left(1-\omega^{2}\right)^{3 / 2}}+\frac{\omega^{2}[\delta(\omega-1)-\delta(\omega+1)]}{\sqrt{1-\omega^{2}}}
$$

is a generalized function. The resulting approximation (33) using $\left(\alpha_{m}, \gamma_{m}\right)$ in Table [4 is presented in Figure 5 and, similarly to approximation (32), it does capture the asymptotic decaying behavior of $J_{1}(x)$.

\subsection{Variations on first order Bessel function of the first kind.}

4.4.1. Approximation using an integral representation through rational approximation. We have already seen that it may be challenging to obtain an expression for $\rho(\omega)$. However, as we have seen it may still be possible to find a rational function $R_{M}(z)$ whose poles and residues provide an appropriate discretization of the measure $\rho(\omega) d \omega$ through the knowledge of $f(x)$ and $g(x)$ so that

$$
f(x) \approx \int_{\Gamma} R_{M}(z) g(z x) d z .
$$


TABLE 4. Table of $\left(\alpha_{m}, \gamma_{m}\right)$ used in approximation $J_{1}(x) \approx \sum_{m=1}^{M} \alpha_{m} \operatorname{sinc}^{\prime}\left(\gamma_{m} x\right)$.

\begin{tabular}{|c|c|c|}
\hline$m$ & $\alpha_{m}$ & $\gamma_{m}$ \\
\hline \hline 1 & -0.001301486125651 & 0.226480239012485 \\
\hline 2 & -0.006606836487331 & 0.381347624932239 \\
\hline 3 & -0.019444628403094 & 0.522032391197420 \\
\hline 4 & -0.045384169021833 & 0.647097634194998 \\
\hline 5 & -0.095302254228288 & 0.754682159355938 \\
\hline 6 & 9.498801270722382 & 1.001443716774511 \\
\hline 7 & -6.317197287638957 & 0.992143409911333 \\
\hline 8 & -1.122223266748518 & 0.962392746785055 \\
\hline 9 & -0.195130288741433 & 0.843495060364425 \\
\hline 10 & -0.422951439023194 & 0.912828994891939 \\
\hline
\end{tabular}
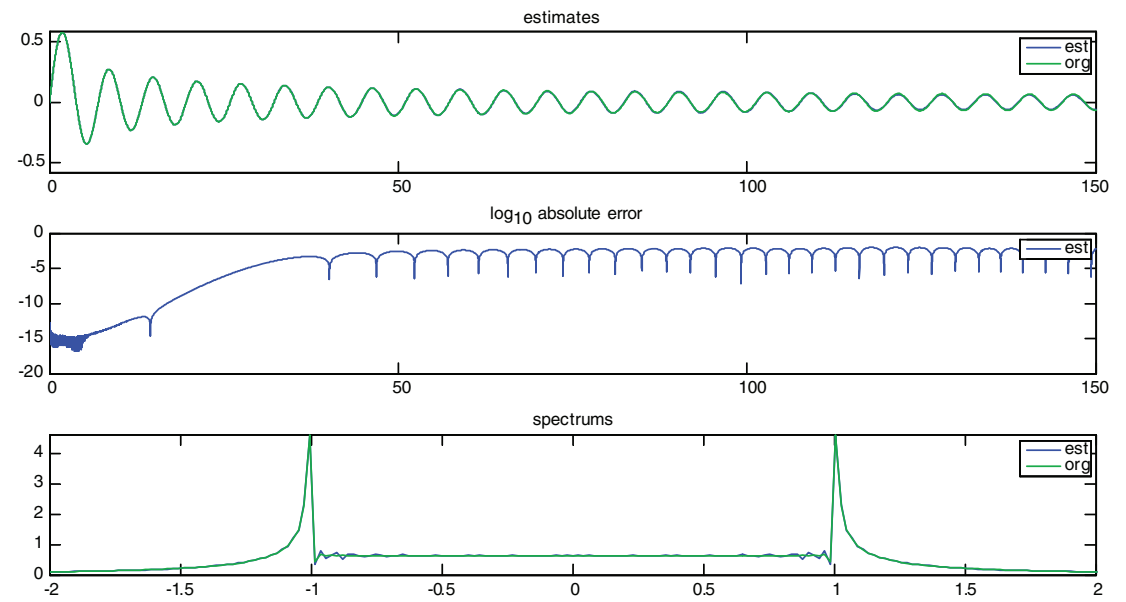

Figure 5. Approximation of the function $J_{1}(x)$ on $[0,150]$ (green) in terms of sum of derivatives of $\operatorname{sincs} J_{1}(x) \approx \sum_{m=1}^{M} \alpha_{m} \operatorname{sinc}^{\prime}\left(\gamma_{m} x\right)$ (blue). The function and its approximation (top), corresponding logarithmic absolute error (middle) and spectra (bottom).

For example, consider $f(x)=J_{1}(x)$ and $g(x)=[1-\cos (x)] / x$. Using the identities

$$
\begin{aligned}
\sin (\omega x) & =\left(\frac{d}{d x} x\right)\left[\frac{1-\cos (\omega x)}{\omega x}\right] \\
& =\left(\frac{d}{d \omega} \omega\right)\left[\frac{1-\cos (\omega x)}{\omega x}\right]
\end{aligned}
$$

and the integral representation for $J_{1}(x)$,

$$
\begin{aligned}
J_{1}(x) & =\frac{2}{\pi} \int_{0}^{1} \frac{\omega}{\sqrt{1-\omega^{2}}} \sin (\omega x) d \omega \\
& =\frac{2}{\pi} \int_{0}^{1} \frac{\omega}{\sqrt{1-\omega^{2}}}\left\{\left(\frac{d}{d \omega} \omega\right)\left[\frac{1-\cos (\omega x)}{\omega x}\right]\right\} d \omega,
\end{aligned}
$$


TABLE 5. Table of $\left(\alpha_{m}, \gamma_{m}\right)$ used in approximation $J_{1}(x) \approx$ $\sum_{m=1}^{M} \alpha_{m}\left(1-\cos \left(\gamma_{m} x\right)\right) /\left(\gamma_{m} x\right)$.

\begin{tabular}{|c|c|c|}
\hline$m$ & $\alpha_{m}$ & $\gamma_{m}$ \\
\hline \hline 1 & -0.014657236787574 & 0.149849719646360 \\
\hline 2 & -0.030791593085749 & 0.295701374119717 \\
\hline 3 & -0.050322558926396 & 0.433883111513795 \\
\hline 4 & -0.076237543720937 & 0.561214541977292 \\
\hline 5 & -0.113915898598797 & 0.675085828291059 \\
\hline 6 & -0.174500782788577 & 0.773516867651912 \\
\hline 7 & 9.820344725976016 & 1.001343018955562 \\
\hline 8 & -6.654085836116296 & 0.992693770137054 \\
\hline 9 & -1.256418697907451 & 0.965062653066941 \\
\hline 10 & -0.526619239685431 & 0.919148643455865 \\
\hline 11 & -0.284975362406715 & 0.855149098929969 \\
\hline
\end{tabular}

one can show that there exists $\rho(\omega)$ such that

$$
J_{1}(x)=\frac{2}{\pi} \int_{0}^{1} \rho(\omega) \frac{1-\cos (\omega x)}{\omega x} d \omega .
$$

However, instead of deriving an explicit representation $\rho(\omega)$, we would like to approximate it in the form (4) for some $\left(\alpha_{m}, \gamma_{m}\right)$. Equating the MacLaurin series decomposition of both sides of (34), implies that $\left(\alpha_{m}, \gamma_{m}\right)$ should satisfy

$$
\sum_{m=1}^{M} \alpha_{m} \gamma_{m}^{2 n-1} \approx \frac{(2 n+2) !}{2^{2 n+1} n !(n+1) !}, \quad n=0,1, \ldots
$$

and

$$
J_{1}(x) \approx \sum_{m=1}^{M} \alpha_{m} \frac{1-\cos \left(\gamma_{m} x\right)}{\gamma_{m} x} .
$$

The resulting approximation (35) using $\left(\alpha_{m}, \gamma_{m}\right)$ in Table 5 is presented in Figure 6 and it does capture the asymptotic decaying behavior of $J_{1}(x)$.

4.4.2. An approximation alternative to derivative. Let us again consider $f(x)=$ $J_{1}(x)$ and, this time, $g(x)=\left[1-J_{0}(x)\right] /(x)$. We would like to obtain an approximation to $J_{1}(x)=-J_{0}^{\prime}(x)$ in the following form:

$$
\begin{aligned}
J_{1}(x) & =-J_{0}^{\prime}(x) \\
& \approx \sum_{m=1}^{2} \alpha_{m} \frac{1-J_{0}\left(\gamma_{m} x\right)}{\gamma_{m} x},
\end{aligned}
$$

where $\rho(x)$ is in the form (4) for some $\left(\alpha_{m}, \gamma_{m}\right)$ satisfying

$$
\sum_{m=1}^{2} \alpha_{m} \gamma_{m}^{2 n+1}=2(n+1), \quad n=0,1, \ldots
$$

and $\gamma_{1}=\overline{\gamma_{2}}=1+\mathrm{i} \gamma$ and $\alpha_{1}=\overline{\alpha_{2}}=1 / 2+\mathrm{i} \alpha$, for some $\gamma, \alpha \in \mathbb{R}$. The approximation (36) is an approximation to differentiation and for $\alpha=-9.804268984562858 \times 10^{7}$ and $\gamma=0.000000005099819$ is presented in Figure 7 . 


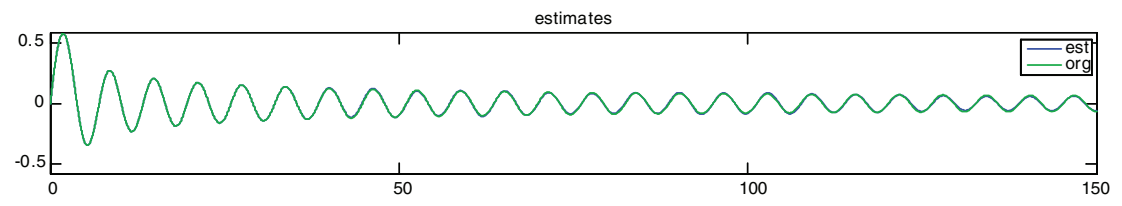

$\log _{10}$ absolute error
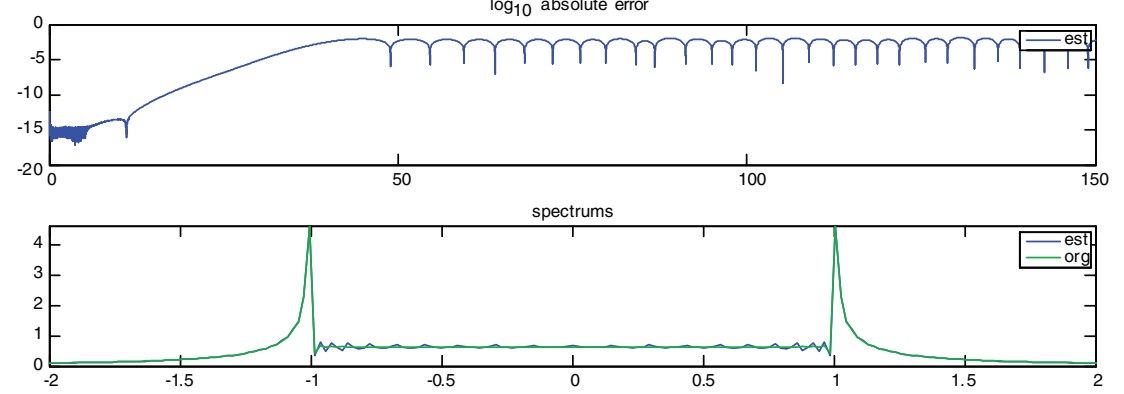

FiguRe 6 . Approximation of the function $J_{1}(x)$ on $[0,150]$ (green) using $g(x)=(1-\cos (x)) / x, J_{1}(x) \approx \sum_{m=1}^{M} \alpha_{m} \frac{1-\cos \left(\gamma_{m} x\right)}{\gamma_{m} x}$ (blue). The function and its approximation (top), corresponding logarithmic absolute error (middle) and spectra (bottom).
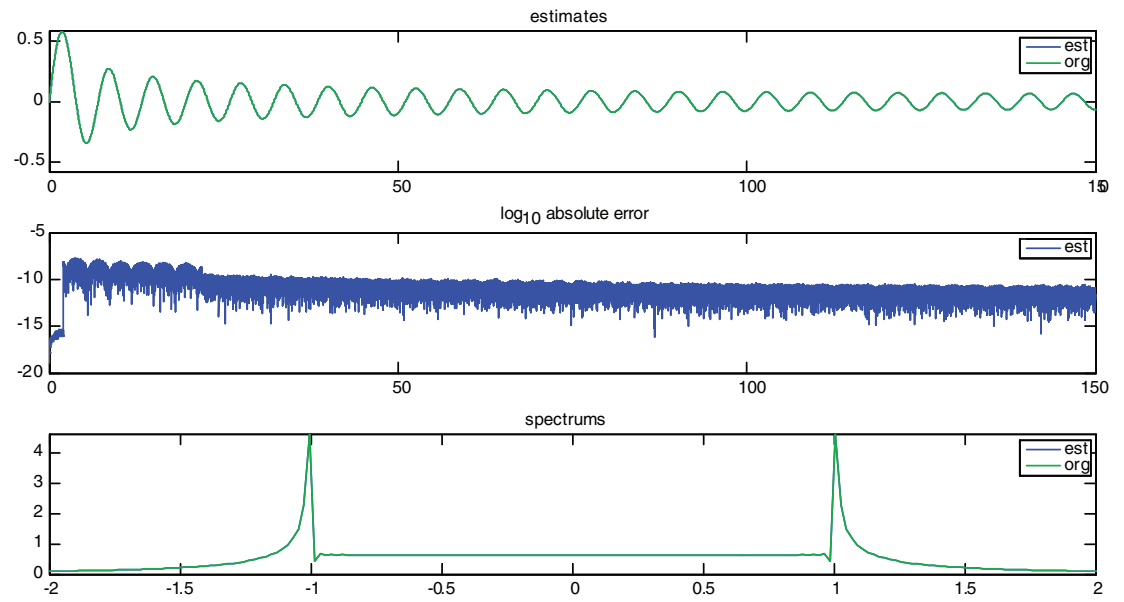

Figure 7. Approximation of the function $J_{1}(x)$ on $[0,150]$ (green) in terms of sum of $\left(1-J_{0}(x)\right) / x: J_{1}(x) \approx \sum_{m=1}^{2} \alpha_{m} \frac{1-J_{0}\left(\gamma_{m} x\right)}{\gamma_{m} x}$ (blue). The function and its approximation (top), corresponding logarithmic absolute error (middle) and spectra (bottom). 
4.5. Band-limited functions. Given a band-limited function $f(x)$ with bandlimit $B>0$, we can write it in terms of sine and cosine as well as sinc and cosinc (see Appendix $\mathrm{C}$ ):

$$
\begin{aligned}
f(x) & =\int_{-B}^{B} F(\omega) \mathrm{e}^{\mathrm{i} \omega x} d \omega \\
& =-\int_{-B}^{B} \omega F^{\prime}(\omega)[\operatorname{sinc}(\omega x)+\mathrm{i} \operatorname{cosinc}(\omega x)] d \omega
\end{aligned}
$$

where $F(\omega)$ is the Fourier transform of $f(x)$. Thus approximations of type (32) and (35) can be extended to band-limited functions:

$$
f(x) \approx \sum_{m=1}^{M_{\alpha}} \alpha_{m} \operatorname{sinc}\left(\gamma_{m} x\right)+\mathrm{i} \sum_{m=1}^{M_{\beta}} \beta_{m} \operatorname{cosinc}\left(\rho_{m} x\right)
$$

where $\left(\alpha_{m}, \gamma_{m}\right)$ and $\left(\beta_{m}, \rho_{m}\right)$ satisfy the moment problems

$$
\begin{gathered}
\sum_{m=1}^{M_{\alpha}} \alpha_{m} \gamma_{m}^{2 n} \approx \frac{f^{(2 n)}(0)}{(2 n) !} \frac{(2 n+1) !}{(-1)^{n}}=(-1)^{n}(2 n+1) f^{(2 n)}(0), \quad n=0,1, \ldots \\
\sum_{m=1}^{M_{\beta}} \beta_{m} \rho_{m}^{2 n+1} \approx \frac{f^{(2 n+1)}}{(2 n+1) !} \frac{(2 n+2) !}{(-1)^{n}}=(-1)^{n}(2 n+2) f^{(2 n+1)}(0), \quad n=0,1, \ldots
\end{gathered}
$$

for some $M_{\alpha}$ and $M_{\beta}$.

Representation of a function in the form of (37) was introduced in discrete setting, analogous to Fourier series, in [2] under the name of sinc-cosinc transform. In this regard, (38) can be regarded as a compact sinc-cosinc representation of a band-limited function. Generalization of Fourier analysis to sinc-cosinc transforms deserves a detailed discussion. Since it is not within the main scope of the current discussion we leave it for a future work.

\section{Conclusions}

We have presented a method of approximation, outlined in Algorithm 1, that is a natural generalization of Padé approximation. The approach provides the flexibility to tailor the choice of approximating function $g$ to both the local and asymptotic properties of $f$, and can achieve highly accurate results. The computational complexity of constructing the approximation is dominated by a singular value decomposition. However, once the approximation is constructed, evaluation of the approximation is linear in the number of evaluation points. The application of our approach is by no means restricted to Bessel functions or band-limited functions, but does appear to perform especially well for integral representations involving oscillatory kernels. For example, similarly accurate approximations can be achieved for the trigonometric integrals such as the sine and cosine integral, as well as Fresnel integrals and the error function. These additional applications of the approach will be exhibited in another work. 


\section{Appendix A. Padé approximation of Zeroth order Bessel function} OF THE FIRST KIND

Since $J_{0}(x)$ is an even function, by Corollary 2, its Padé approximation is given by

$$
J_{0}(x) \approx \sum_{m=1}^{M} \frac{\alpha_{m}}{1-\gamma_{m} x^{2}}
$$

where $\left(\alpha_{m}, \gamma_{m}\right)$ satisfies the moment problem

$$
\frac{J_{0}^{(2 n)}(0)}{(2 n) !}=\frac{(-1)^{n}}{\left(2^{n} n !\right)^{2}} \approx \sum_{m=1}^{M} \alpha_{m} \gamma_{m}^{n}
$$

for some fixed $M$. For $M=11$, the Padé approximation of type [21/22] is obtained by using $\left(\alpha_{m}, \gamma_{m}\right)$ in Table 6 and is presented in Figure 8 ,

TABLE 6. $\left(\alpha_{m}, \gamma_{m}\right)$ used in approximation $J_{0}(x) \approx \sum_{m=1}^{M} \alpha_{m} /\left(1-\gamma_{m} x^{2}\right)$.

\begin{tabular}{|c|c|c|}
\hline$m$ & $\alpha_{m} \times 10^{-8}$ & $\gamma_{m}$ \\
\hline \hline 1 & $-0.000614788084697+0.001585875079785 \mathrm{i}$ & $0.000291575752811+0.001291852140716 \mathrm{i}$ \\
\hline 2 & $-0.000614788085235-0.001585875079374 \mathrm{i}$ & $0.000291575752811-0.001291852140716 \mathrm{i}$ \\
\hline 3 & $-0.033915222472068-0.014392877781962 \mathrm{i}$ & $-0.000293512765097+0.001410757164885 \mathrm{i}$ \\
\hline 4 & $-0.033915222463937+0.014392877792491 \mathrm{i}$ & $-0.000293512765097-0.001410757164885 \mathrm{i}$ \\
\hline 5 & $0.012146382836224+0.982497802051191 \mathrm{i}$ & $-0.001250873778915+0.000958068730542 \mathrm{i}$ \\
\hline 6 & $0.012146382529713-0.982497802005579 \mathrm{i}$ & $-0.001250873778915-0.000958068730542 \mathrm{i}$ \\
\hline 7 & $-1.483851002320522-1.406678773400033 \mathrm{i}$ & $-0.001523716898135+0.000511568465477 \mathrm{i}$ \\
\hline 8 & $-1.483851001858117+1.406678773810973 \mathrm{i}$ & $-0.001523716898135-0.000511568465477 \mathrm{i}$ \\
\hline 9 & 2.594611617209278 & -0.001617733593762 \\
\hline 10 & $0.208928826334127-0.171620380199425 \mathrm{i}$ & $-0.000826323555787+0.001277342361164 \mathrm{i}$ \\
\hline 11 & $0.208928826375233+0.171620380118650 \mathrm{i}$ & $-0.000826323555787-0.001277342361164 \mathrm{i}$ \\
\hline
\end{tabular}
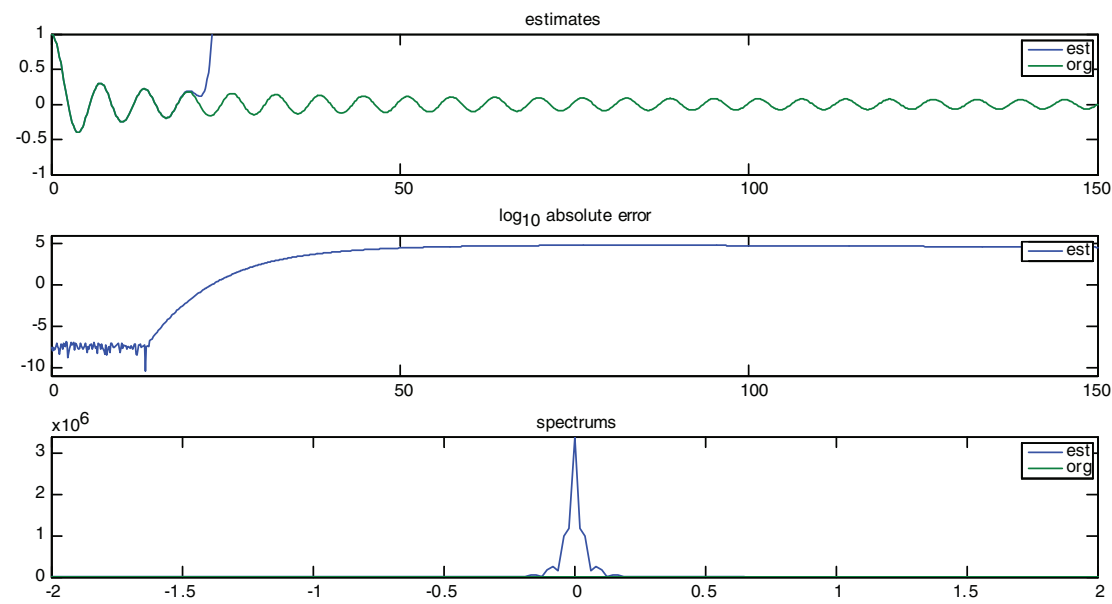

Figure 8. Padé approximation (blue) of the Bessel function $J_{0}(x)$ on $[0,150]$ (green). The function and its approximation (top), corresponding logarithmic absolute error (middle) and spectra (bottom). 


\section{Appendix B. Bessel Function in the integral Form

$$
J_{0}(x)=\int_{0}^{1} \rho(\omega) \operatorname{sinc}(\omega x) d \omega
$$

Using the orthogonality property of $\operatorname{sinc}(\omega x)$

$$
x^{\prime 2} \int_{0}^{\infty} \operatorname{sinc}(\omega x) \operatorname{sinc}\left(\omega x^{\prime}\right) \omega^{2} d \omega=\delta\left(x-x^{\prime}\right),
$$

one can write $\rho(\omega)$ by

$$
\begin{aligned}
\rho(\omega) & =\omega^{2} \int_{0}^{\infty} \operatorname{sinc}(\omega x) J_{0}(x) x^{2} d x \\
& =\omega^{2} \int_{0}^{\infty} \frac{\sin (\omega x)}{\omega} J_{0}(x) x d x \\
& =\omega \int_{0}^{\infty} \sin (\omega x) J_{0}(x) x d x \\
& =-\omega \frac{d}{d \omega}\left[\int_{0}^{\infty} \cos (\omega x) J_{0}(x) d x\right] \\
& =-\omega \frac{d}{d \omega}\left[\frac{\chi_{[-1,1]}(\omega)}{\sqrt{1-\omega^{2}}}\right] \\
& =-\omega\left[\frac{\delta(\omega+1)-\delta(\omega-1)}{\sqrt{1-\omega^{2}}}+\frac{\omega \chi_{[-1,1]}(\omega)}{\left(1-\omega^{2}\right)^{3 / 2}}\right] \\
& =\frac{\omega[\delta(\omega-1)-\delta(\omega+1)]}{\sqrt{1-\omega^{2}}}-\frac{\omega^{2} \chi_{[-1,1]}(\omega)}{\left(1-\omega^{2}\right)^{3 / 2}} .
\end{aligned}
$$

\section{Appendix C. BAnd-Limited Functions}

Let $f(x)$ be defined in terms of its Fourier transform $F(\omega)$

$$
f(x)=\int_{-\infty}^{\infty} F(\omega) \mathrm{e}^{\mathrm{i} \omega x} d \omega
$$

where

$$
F(\omega)=\frac{1}{2 \pi} \int_{-\infty}^{\infty} f(x) \mathrm{e}^{-\mathrm{i} \omega x} d x .
$$

We say that $f(x)$ is band-limited with band-limit $B>0$ if $F(\omega)=0$, for $|\omega|<B$. Let $f(x)$ be band-limited with band-limit $B$ and $F(\omega)$ be differentiable for $|\omega|<B$. Then the derivatives of $F(\omega)$ are also band-limited with band-limit $B$. We then have the following lemma.

Lemma 1. Let $f(x)$ be a band-limited function with band-limit $B>0$, whose Fourier transform $F(\omega)$ is differentiable. Then

$$
f(x)=-\int_{-B}^{B} \omega F^{\prime}(\omega)[\operatorname{sinc}(\omega x)+\mathrm{i} \operatorname{cosinc}(\omega x)] d \omega .
$$

Proof. Decomposing $F(\omega)=F_{\text {even }}(\omega)+F_{\text {odd }}(\omega)$ into even and odd parts

$$
\begin{aligned}
F_{\text {even }}(\omega) & =\frac{F(\omega)+F(-\omega)}{2}, \\
F_{\text {odd }}(\omega) & =\frac{F(\omega)-F(-\omega)}{2},
\end{aligned}
$$


we decompose (39) as

$$
\begin{aligned}
f(x) & =\int_{-\infty}^{\infty} F(\omega) \cos (\omega x) d \omega+\mathrm{i} \int_{-\infty}^{\infty} F(\omega) \sin (\omega x) d \omega \\
& =\int_{-\infty}^{\infty} F_{\text {even }}(\omega) \cos (\omega x) d \omega+\mathrm{i} \int_{-\infty}^{\infty} F_{\text {odd }}(\omega) \sin (\omega x) d \omega \\
& =2 \int_{0}^{\infty} F_{\text {even }}(\omega) \cos (\omega x) d \omega+2 \mathrm{i} \int_{0}^{\infty} F_{\text {odd }}(\omega) \sin (\omega x) d \omega .
\end{aligned}
$$

Performing integration by parts on the integrals, we obtain

$$
\begin{aligned}
\int_{0}^{\infty} F_{\text {even }}(\omega) \cos (\omega x) d \omega & =\left.\left[\omega F_{\text {even }}(\omega) \frac{\sin (\omega x)}{\omega x}\right]\right|_{\omega=0} ^{\omega=\infty}-\int_{0}^{\infty} F_{\text {even }}^{\prime}(\omega) \frac{\sin (\omega x)}{x} d \omega \\
& =-\int_{0}^{\infty} \omega F_{\text {even }}^{\prime}(\omega) \operatorname{sinc}(\omega x) d \omega \\
& =-\frac{1}{2} \int_{-\infty}^{\infty} \omega F^{\prime}(\omega) \operatorname{sinc}(\omega x) d \omega
\end{aligned}
$$

and

$$
\begin{aligned}
\int_{0}^{\infty} F_{\text {odd }}(\omega) \sin (\omega x) d \omega & =-\left.\left[F_{\text {odd }}(\omega) \frac{\cos (\omega x)}{x}\right]\right|_{\omega=0} ^{\omega=\infty}+\int_{0}^{\infty} F_{\text {odd }}^{\prime}(\omega) \frac{\cos (\omega x)}{\omega x} d \omega \\
& =F_{\text {odd }}(\omega) \frac{1}{x}+\int_{0}^{\infty} F_{\text {odd }}^{\prime}(\omega) \frac{\cos (\omega x)}{x} d \omega \\
& =-\frac{1}{x} \int_{0}^{\infty} F_{\text {odd }}^{\prime}(\omega) d \omega+\int_{0}^{\infty} F_{\text {odd }}^{\prime}(\omega) \frac{\cos (\omega x)}{x} d \omega \\
& =-\int_{0}^{\infty} F_{\text {odd }}^{\prime}(\omega) \frac{1-\cos (\omega x)}{x} d \omega \\
& =-\int_{0}^{\infty} \omega F_{\text {odd }}^{\prime}(\omega) \operatorname{cosinc}(\omega x) d \omega \\
& =-\frac{1}{2} \int_{-\infty}^{\infty} \omega F^{\prime}(\omega) \operatorname{cosinc}(\omega x) d \omega
\end{aligned}
$$

where the cosinc is defined by

$$
\operatorname{cosinc}(x)=\frac{1-\cos (x)}{x} .
$$

Substituting (43) and (44) into (42), we obtain

$$
\begin{aligned}
f(x) & =2 \int_{0}^{\infty} F_{\text {even }}(\omega) \cos (\omega x) d \omega+2 \mathrm{i} \int_{0}^{\infty} F_{\text {odd }}(\omega) \sin (\omega x) d \omega \\
& =-2 \int_{0}^{\infty} \omega F_{\text {even }}^{\prime}(\omega) \operatorname{sinc}(\omega x) d \omega-2 \mathrm{i} \int_{0}^{\infty} \omega F_{\text {odd }}^{\prime}(\omega) \operatorname{cosinc}(\omega x) d \omega \\
& =-\int_{-\infty}^{\infty} \omega F^{\prime}(\omega)[\operatorname{sinc}(\omega x)+\mathrm{i} \operatorname{cosinc}(\omega x)] d \omega,
\end{aligned}
$$

which, due to the band-limited nature of $F(\omega)$, leads to (41). 


\section{ACKNOWLEDGMENTS}

We would like to take the opportunity to thank Lucas Monzon, Paul Childs and Aimè Fournier for fruitful discussions and comments. We thank the management of Schlumberger for permission to publish this work.

\section{REFERENCES}

[1] Milton Abramowitz and Irene A. Stegun (eds.), Handbook of Mathematical Functions with Formulas, Graphs, and Mathematical tables, Dover Publications Inc., New York, 1992. Reprint of the 1972 edition. MR.1225604 (94b:00012)

[2] Robert $\mathrm{F}$ Abramson, The sinc and cosinc transform, Electromagnetic Compatibility, IEEE Transactions on (1977), no. 2, 88-94.

[3] Lars V. Ahlfors, Complex Analysis: An Introduction to the Theory of Analytic Functions of One Complex Variable, 3rd ed., International Series in Pure and Applied Mathematics, McGraw-Hill Book Co., New York, 1978. MR.510197 (80c:30001)

[4] D.E. Amos, A subroutine package for bessel functions of a complex argument and nonnegative order, Sandia National Laboratory Report SAND85-1018, Sandia National Laboratory, May 1985.

[5] D. E. Amos, Algorithm 644: a portable package for Bessel functions of a complex argument and nonnegative order, ACM Trans. Math. Software 12 (1986), no. 3, 265-273, DOI 10.1145/7921.214331. MR889069

[6] Athanasios C. Antoulas, Approximation of Large-Scale Dynamical Systems, Advances in Design and Control, vol. 6, Society for Industrial and Applied Mathematics (SIAM), Philadelphia, PA, 2005. With a foreword by Jan C. Willems. MR2155615 (2006c:93001)

[7] George A. Baker Jr., Essentials of Padé Approximants, Academic Press [A subsidiary of Harcourt Brace Jovanovich, Publishers], New York-London, 1975. MR0454459 (56 \#12710)

[8] George A. Baker Jr. and Peter Graves-Morris, Padé Approximants, 2nd ed., Encyclopedia of Mathematics and its Applications, vol. 59, Cambridge University Press, Cambridge, 1996. MR.1383091 (97h:41001)

[9] Gregory Beylkin and Lucas Monzón, On approximation of functions by exponential sums, Appl. Comput. Harmon. Anal. 19 (2005), no. 1, 17-48, DOI 10.1016/j.acha.2005.01.003. MR:2147060 (2006h:65012)

[10] Gregory Beylkin and Lucas Monzón, Approximation by exponential sums revisited, Appl. Comput. Harmon. Anal. 28 (2010), no. 2, 131-149, DOI 10.1016/j.acha.2009.08.011. MR2595881 (2011e:41032)

[11] C. F. Gauss, Methodus nova integralium valores per approximationem inveniendi, Comment. Soc. Reg. Scient. Gotting. Recent. (1814).

[12] Walter Gautschi, Construction of Gauss-Christoffel quadrature formulas, Math. Comp. 22 (1968), 251-270. MR0228171 (37 \#3755)

[13] R. E. Kalman, On partial realizations, transfer functions, and canonical forms, Acta Polytech. Scand. Math. Comput. Sci. Ser. 31 (1979), 9-32. Topics in systems theory. MR557691 (80k:93022)

[14] Rudolf Emil Kalman, Mathematical description of linear dynamical systems, Journal of the Society for Industrial \& Applied Mathematics, Series A: Control 1 (1963), no. 2, 152-192.

[15] R. E. Kalman, Advanced theory of linear systems, Topics in Mathematical System Theory, McGraw-Hill, New York, 1969, pp. 237-339. MR0286517 (44 \#3726)

[16] Sun-Yuan Kung, A new identification and model reduction algorithm via singular value decomposition, Proc. 12th Asilomar Conf. Circuits, Syst. Comput., Pacific Grove, CA, 1978, pp. $705-714$.

[17] Sun Yuan Kung and David W. Lin, A state-space formulation for optimal Hankelnorm approximations, IEEE Trans. Automat. Control 26 (1981), no. 4, 942-946, DOI 10.1109/TAC.1981.1102736. MR635856 (84a:93017b)

[18] A. J. Mayo and A. C. Antoulas, A framework for the solution of the generalized realization problem, Linear Algebra Appl. 425 (2007), no. 2-3, 634-662, DOI 10.1016/j.laa.2007.03.008. MR2343060 (2008e:93028) 
[19] I. M. Gel'fand and G. E. Shilov, Generalized Functions. Vol. 1, Academic Press [Harcourt Brace Jovanovich Publishers], New York, 1964 [1977]. Properties and operations; Translated from the Russian by Eugene Saletan. MR0435831 (55 \#8786a)

[20] Leonard M. Silverman, Realization of linear dynamical systems, IEEE Trans. Automatic Control AC-16 (1971), 554-567. MR0307749 (46 \#6869)

Schlumberger, 3750 Briar Park Dr., Houston, Texas 77042

E-mail address: cyarman@slb.com

Schlumberger, 3750 Briar Park Dr., Houston, Texas 77042

E-mail address: GFlagg@slb.com 